\title{
Cálculo de las propiedades geotécnicas a partir de las velocidades transversales obtenidas en la sísmica de refracción. Estudio de caso en la ciudad de Luanda (Angola)
}

\author{
Maria Amélia Pereira de Barros da Silva ${ }^{1 *}$; Héctor Manuel Fernández ${ }^{2}$ J João Júnior Baptista ${ }^{1}$; Gabriela \\ Jeremias Pereira Teixeira-Pires ${ }^{3}$
}

DOI: http://dx.doi.org/10.18273/revbol.v40n2-2018006 @c (1)

Forma de citar: De Barros da Silva, M.A.P., Fernández, H.M., Baptista, J.Jr., y Teixeira-Pires, G.J.P. (2018). Cálculo de las propiedades geotécnicas a partir de las velocidades transversales obtenidas en la sísmica de refracción. Estudio de caso en la ciudad de Luanda (Angola). Boletín de Geología, 40(2), 101-112. DOI: $10.18273 /$ revbol.v40n2-2018006.

\section{RESUMEN}

La sísmica de refracción es un método de geofísica de prospección, que estudia las respuestas del terreno cuando se propaga a través de él una onda de compresión (onda P) producida por medios mecánicos en la superficie. El parámetro físico que se utiliza es la velocidad de propagación de la onda Vp que viaja a través de los materiales del subsuelo en función de la densidad del mismo. Tal parámetro aportará información de las características geomecanicas de la zona en la que se aplican. Los índices medidos mantienen correlaciones con la naturaleza geológica de los diversos horizontes, pudiéndose aún conocer sus respectivas profundidades y espesores. Este trabajo tiene como objectivo presentar un procedimiento para la representación de los parámetros geotécnicos, estimados por el método de refracción sísmica. Las velocidades longitudinales son obtenidas y se correlacionan con los valores de la resistencia a la penetración estandar $(\mathrm{N})$ calculados por los ensayos homónimos de SPT. Los parámetros dinámicos se presentan en secciones 2D para conseguir una mejor representación e interpretación, siendo capaz de caracterizar continuamente todo el área desde el punto de vista geotécnico. El área de estudio está localizada en los suelos areno - limo - arcillosos de la ciudad de Luanda (Angola).

Palabras clave: Refracción sísmica; SPT; Parámetros dinámicos; Cartografía geotécnica.

\section{Determination of geothecnical properties by using seismic refraction velocities. A case study in Luanda (Angola)}

\begin{abstract}
Seismic refraction is a prospecting geophysical method, which measures the terrain response in reaction to a $\mathrm{P}$ compression wave, mechanically produced at the surface and propagating across. The physical parameter in use, in the case, is the wave propagation velocity, $\mathrm{Vp}$, which travel across the subsurface rock material and reflects to the surface in function of the material's density it finds on its way down. That way, indexes associated to the parameter will measure and reveal the geomechanic characteristics of the terrain under study. Those indexes, whilst keeping some correlations with the geological nature of the rock in different horizons, allow to infer also their depths and thickness. The present study has the scope of showing a methodology to map geotechnical parameters using seismic reflection, and it has been noticed that the longitudinal velocities also computed are correlated with penetration resistance values, measured by Standard Penetration Tests (SPT). The dynamic parameters were chosen to be presented in 2D maps, in order to give either, a better representation of their characteristics, as well to allow for a continuous geotechnical interpretation over the entire area of study. The latter, is an area of silty/sandy soils located in the city of Luanda (Angola).
\end{abstract}

Keywords: Seismic refraction; SPT; Dynamic parameters; Geotechnical mapping.

\footnotetext{
${ }^{1}$ Geofísica, Faculdad de Ciencias, Universidad Agostinho Neto, Luanda, Angola. (*) mely-barros@hotmail.com; Joao.baptista@segeo.org

2Universidad Tecnólogica de La Habana “José António Echeverría”, Marianao, La Habana, Cuba. hector@civil.cujae.edu.cu

${ }^{3}$ Geología, Faculdad de Ciencias, Universidad Agostinho Neto, Luanda, Angola. gabrielapires@hotmail.com
} 


\section{INTRODUCCIÓN}

Los métodos geofísicos han sido utilizados desde algunos años en las prospeciones geotécnicas y sus resultados han mejorado substancialmente estas investigaciones. Ejemplo de eso, son los trabajos de Luna y Jadi (2000), Naranjo y Dranichnikov (2012) y Nabil y Al-Heety (2014) por solo mencionar algunos de los consultados para esta investigación. La principal dificultad que se observa es en la representación de sus resultados que muchas veces deben ser presentados a ingenieros $u$ otros investigadores que no entienden o desconocen en muchos casos el lenguaje de las Geociencias. Por otro lado, los parámetros físicomecánicos calculados se presentan en tablas o se ofrecen sus valores para las diferentes capas sin reflejar la continuidad en la representación que dan estos métodos. Debido a lo anterior, el objetivo principal de este trabajo es el de ofrecer un procedimiento para la representación de los parámetros geotécnicos estimados por la sísmica de refracción, tomando como base los datos sísmicos medidos en un área de la ciudad de Luanda, Angola.

\section{CONTEXTO GEOLÓGICO}

\section{Características geológicas del área de estudio}

El terreno para esta campaña de prospección se sitúa en el barrio Proyecto Nueva Vida, en Luanda (FIGURA 1).

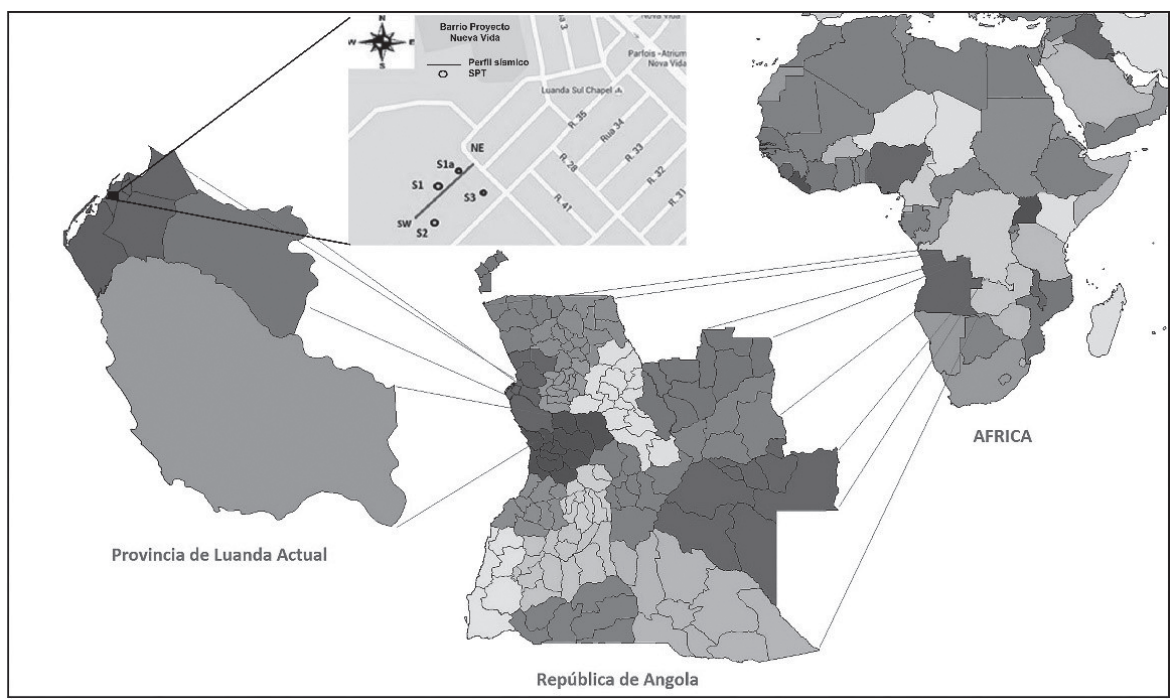

FIGURA 1. Localización del área de estudio y líneas sísmicas analizadas (adaptado de Google Map).

De acuerdo con la carta litológica, en la zona de Luanda aflora la parte superior de una potente sucesión Mesozoica y Terciaria que abarca en profundidad un máximo de $4000 \mathrm{~m}$ de espesor conformando tres cuencas sedimentarias siendo la más importante la cuenca del Kwanza que constituye gran parte de la franja de costa de Angola. La secuencia estratigráfica que se apoya sobre el basamento Pre-cambrico viene expuesta de forma sintética en la FIGURA 2.

\section{Litología}

En la región de Luanda se distinguen dos unidades litológicas: las someras, que corresponden a las unidades recientes del Pleistoceno y las del substrato del Plioceno y del Mioceno (FIGURA 2).

Las unidades recientes incluyen los materiales no consolidados de origen antrópico (A), los aluviones, constituidos por materiales heterogéneos y los sedimentos fluvio-lacustres (Al), las arenas de playa (ap) que constituyen las islas y cordones litorales, los depósitos de vertiente (a3) y conos de deyección (a2), los aluviones recientes (a1), que están asociados a las líneas de flujo superficial de agua, y la unidad de las arcillas negras o arcillas del Cazenga (Q3). Las formaciones Quelo con dos unidades, arenas rojas (Q2) y arenas grises (Q1) constituidas esencialmente por arenas de edad Pleistoceno, se incluyen también en las unidades someras. Las unidades del substrato corresponden a la formación Luanda (p1) del Plioceno, constituida por materiales no consolidados arenas, limos y arcillas y materiales consolidados de arenas y la formación Cacuaco (m1c), constituida por carbonatos descompuestos y calizas (FIGURA 2).

En el área de Luanda apenas afloran terrenos desde el Mioceno Inferior. Las formaciones reconocidas son, de la base para el tope, Quifangondo (m1), Cacuaco 
(m1c), Luanda (p1), Arenas cenizas (Q1), formación Quelo o Arena de Muceque (Q2) y la unidad informal de las Arcillas negras (Q3), o Formación del Cazenga. Sobre este paquete geológico se asientan otras formaciones del Holoceno, entre las que pueden ser nombradas están las arenas de dunas y de playa y depósitos actuales diversos (Teixeira Pires, 2006).
El objeto de estudio de esta investigación son los terrenos transportados del Pleistoceno y Plioceno de las formaciones Quelo y Luanda, respetivamente que constituyen las unidades geotécnicas de interés en esta área. Como característica propia de esta región se destaca la no presencia de un sustrato rocoso, por lo que se estudiaran sedimentos areno limo arcillosos que se compactan con la profundidad.

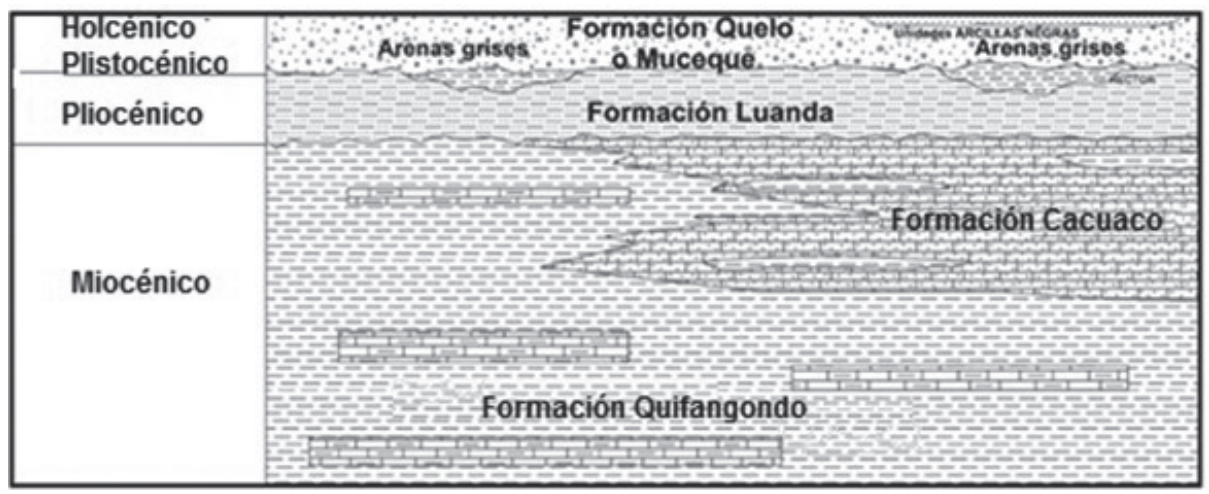

FIGURA 2. Esquema geológico estratigráfico de la ciudad de Luanda, Angola (modificado de Tecnasol, 2007).

\section{METODOLOGÍA}

\section{Campaña de prospección geológico - geotécnica}

El trabajo consistió en la perforación de sondeos ejecutados con un equipo de percusión, acompañados de la realización de ensayos de penetración dinámica tipo SPT cada $1,5 \mathrm{~m}$. Las prospecciones fueron dadas por terminadas despues de haber alcanzado un mínimo de 15 metros de profundidad y despues de lograrse tres valores de $\mathrm{N}_{\mathrm{SPT}}>60$ golpes, a excepción de la prospección S1, donde no se consiguió proseguir la perforación debido a la naturaleza de las litologias encontradas.

Los diámetros de la perforación utilizada fueron de 8" у 6"(Ø 203,20 mm у Ø $152,40 \mathrm{~mm})$, siendo los pozos protegidos por tubería de revestimiento de igual diámetro, conforme al propio método de perforación (Tecnasol, 2007).

Los ensayos de penetración dinámica, tipo SPT, tienen como objetivo la determinación de la resistencia del suelo a la penetración de un muestreador estandardizado, al mismo tiempo se obtienen muestras representativas. Son realizados dentro de los pozos de prospección a profundidades definidas, y siempre que ocurra un cambio de litologia. Los resultados de los cuatro sondeos realizados, se pueden observar en la TABLA 1 y representados en un perfil en la FIGURA 3.

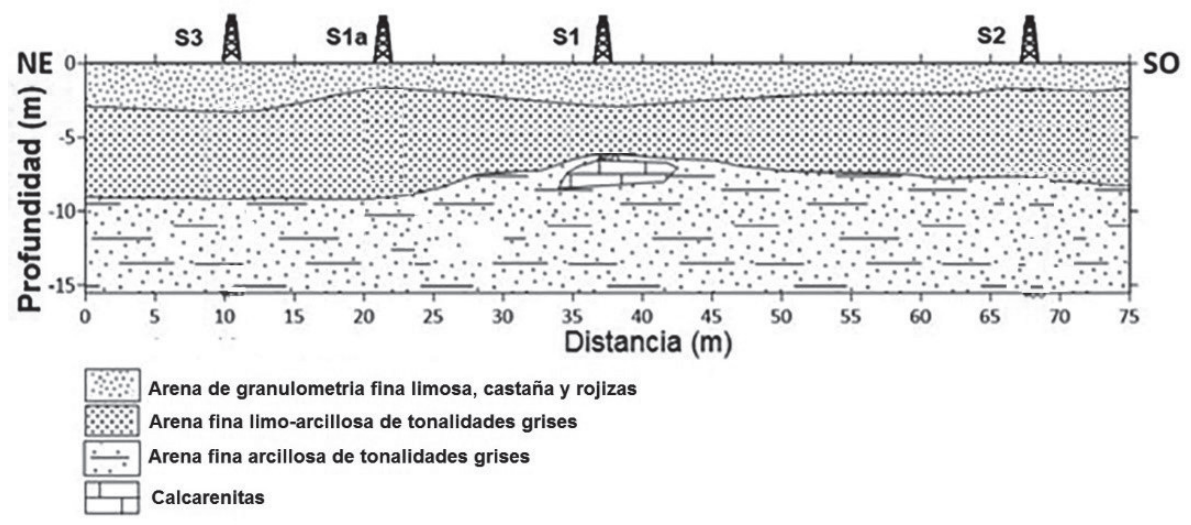

FIGURA 3. Perfil litológico obtenido con los ensayos de SPT de campo. 
Cálculo de las propiedades geotécnicas a partir de las velocidades transversales obtenidas en la sísmica de refracción. Estudio de caso en la ciudad de Luanda (Angola)

TABLA 1. Resultados de los ensayos de SPT.

\begin{tabular}{|c|c|c|c|c|c|c|c|c|}
\hline \multirow{2}{*}{$\begin{array}{l}\text { Profundidad } \\
(\mathrm{m})\end{array}$} & S3 & \multirow{2}{*}{ Litología } & S1a & \multirow{2}{*}{ Litología } & S1 & \multirow{2}{*}{ Litología } & S2 & \multirow{2}{*}{ Litología } \\
\hline & $\mathrm{N}_{\mathrm{SPT}}$ & & $\mathrm{N}_{\mathrm{SPT}}$ & & $\mathrm{N}_{\mathrm{SPT}}$ & & $\mathrm{N}_{\mathrm{SPT}}$ & \\
\hline 2 & 22 & \multirow{2}{*}{$\begin{array}{l}\text { Arena fina } \\
\text { limosas } \\
\text { castaña } \\
\text { rojiza }\end{array}$} & 38 & $\begin{array}{l}\text { Arena de } \\
\text { granulometría } \\
\text { fina limosa, } \\
\text { castañas y } \\
\text { rojizas }\end{array}$ & 18 & \multirow[b]{2}{*}{$\begin{array}{l}\text { Arena fina } \\
\text { castaña y } \\
\text { rojiza }\end{array}$} & 52 & $\begin{array}{l}\text { Arena fina } \\
\text { limosas } \\
\text { castaña y } \\
\text { rojiza }\end{array}$ \\
\hline 3,5 & 27 & & 42 & $\begin{array}{l}\text { Arena limo- } \\
\text { arcillosa de } \\
\text { granulometría } \\
\text { fina de } \\
\text { tonalidades } \\
\text { blancas, grises } \\
\text { y amarillas }\end{array}$ & 21 & & 43 & \multirow{3}{*}{$\begin{array}{c}\text { Arena } \\
\text { limosa de } \\
\text { tonalidades } \\
\text { grises }\end{array}$} \\
\hline 5 & 32 & \multirow{3}{*}{$\begin{array}{l}\text { Arena } \\
\text { fina limo- } \\
\text { arcillosa de } \\
\text { tonalidades } \\
\quad \text { grises }\end{array}$} & 48 & $\begin{array}{c}\text { Arena de } \\
\text { granulometría } \\
\text { gruesa, de } \\
\text { tonalidades } \\
\text { blancas }\end{array}$ & 38 & $\begin{array}{c}\text { Arena fina } \\
\text { limo-arcillosa } \\
\text { blanquecina }\end{array}$ & 54 & \\
\hline 6,5 & 40 & & & \multirow{6}{*}{$\begin{array}{l}\text { Arena limo- } \\
\text { arcillosa de } \\
\text { granulometría } \\
\text { fina de } \\
\text { tonalidades } \\
\text { blancas, grises } \\
\text { y amarillas }\end{array}$} & & $\begin{array}{c}\text { Calcarenitas } \\
\text { brechosas de } \\
\text { tonalidades } \\
\text { amarillas } \\
\text { pasando } \\
\text { a negro, } \\
\text { recuperado } \\
\text { como } \\
\text { fragmentos }\end{array}$ & 54 & \\
\hline 8 & 50 & & 50 & & & & 60 & $\begin{array}{c}\text { Arena fina de } \\
\text { tonalidades } \\
\text { blancas }\end{array}$ \\
\hline 9,5 & 60 & \multirow{5}{*}{$\begin{array}{l}\text { Arena fina } \\
\text { arcillosa de } \\
\text { tonalidades } \\
\text { grises }\end{array}$} & 60 & & & & 60 & \multirow{5}{*}{$\begin{array}{l}\text { Arena limo- } \\
\text { arcillosa de } \\
\text { tonalidades } \\
\text { grises }\end{array}$} \\
\hline 11 & 60 & & 60 & & & & 60 & \\
\hline 12,5 & 60 & & 60 & & & & 60 & \\
\hline 14 & 60 & & 60 & & & & 60 & \\
\hline 15,5 & 60 & & & & & & 60 & \\
\hline
\end{tabular}

\section{Adquisición geofísica}

\section{Sísmica de refracción}

La refracción sísmica se basa en el estudio de las ondas elásticas, para determinar en terminos de velocidad y espesor, la disposición de las capas en el subsuelo, siendo posible detectar, con un grado elevado de fiabilidad estructuras geológicas en el subsuelo de un área (Lankston, 1989).

Según Sacasas (2012), la refracción sísmica capta la componente refractada de una onda entre dos medios con diferentes propiedades elásticas a lo largo de una serie de geófonos colocados en la superficie del suelo. La onda en el subsuelo es generada por una fuente impulsiva a partir de impactos directos en el suelo o por explosivos. Se logra registrar el movimiento de las ondas desde la fuente hasta los receptores a través de un sismógrafo, con diferentes arreglos de geófonos. Es de particular interés, para este método, las primeras entradas (ondas directas y ondas P refractada críticamente). Con las informaciones suministradas por esas ondas se construyen los gráficos tiempo-distancia, a partir de los cuales se realiza la interpretación y 
se obtienen las velocidades y los espesores de las diferentes capas del corte geológico.

\section{Adquisición y tratamiento de los datos sísmicos}

\section{Metodología de adquisición}

Para la adquisición de los datos fue utilizado un sismógrafo de la Geometrics, modelo Geode de 24 canales y geófonos de 14 ó $40 \mathrm{~Hz}$ siguiendo los procedimientos de la Norma ASTM D5777-95. La distancia entre los geófonos fue igual a 2 metros, generando una línea sísmica de 46 metros de largo, donde el primer geófono es considerado de referencia, se queda en la posición cero. La fuente de energía fue un martillo de aproximadamente 10 kilogramos con un trigger de contacto acoplado. Para aumentar el área de impacto entre el martillo y el suelo una chapa metálica fue usada, generando un frente de ondas mecánicas inicial mayor. Los puntos de impacto fueron dispuestos, con relación al geófono de referencia, en las posiciones: $-12 ;-2 ; 23 ; 48 ; 58$ metros (FIGURA 4). Cada punto de impacto fue caracterizado por el apilamiento (suma) de una secuencia de 30 golpes, lo que permitió atenuar los ruidos y aumentar la resolución.

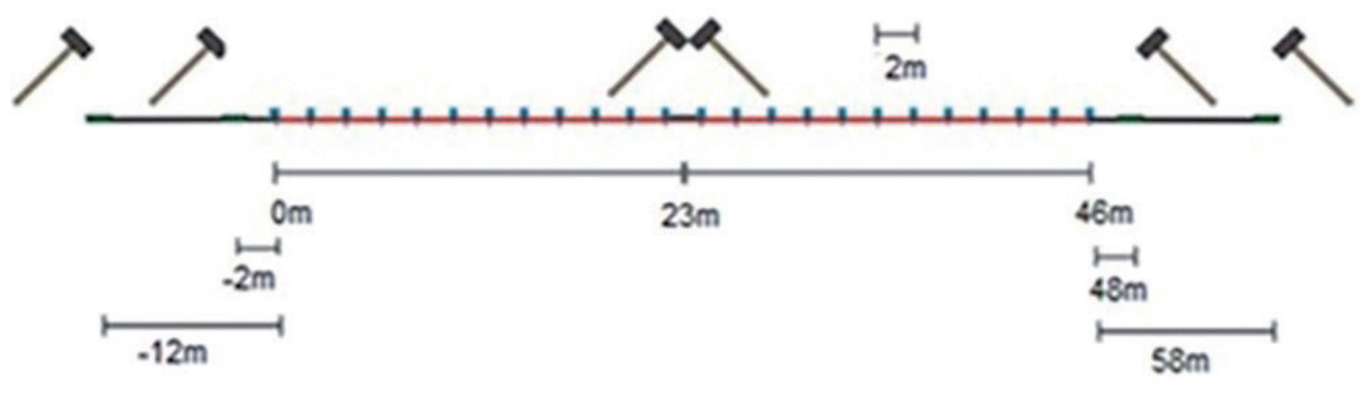

FIGURA 4. Configuración del arreglo utilizado en el levantamiento de los datos (modificado de Araújo y Peres-Rocha, 2010).

Durante el proceso de adquisición de los datos se realizó un pre-procesamiento en el campo lo que permitió controlar la calidad de los datos que estaban siendo adquiridos. Para ejecución de este procedimiento fue usado el SIPQC que es un programa de interpretación interactivo confeccionado por la United States Geological Survey y la versión QC y que está incorporado en el software SCS (acquisition control software). El procedimiento obedeció a tres etapas: marcación de las primeras llegadas, generación del gráfico tiempo-distancia e inversión para crear el modelo de profundidades. La primera etapa en el análisis de los datos de sísmica de refracción consiste en la identificación de las primeras llegadas de la energía sísmica en cada traza. La FIGURA 5, ilustra el sismograma referido a la línea sísmica de Nueva Vida, generado con el impacto en el punto central (23 metros), donde fueron hechas marcaciones de las primeras llegadas de las ondas refractadas. La marcación de las primeras llegadas de todos los puntos de impacto suministró el gráfico del tiempo-distancia ilustrado en la FIGURA 6. Después de la marcación de las primeras llegadas y la generación del gráfico del tiempo-distancia se le realizó el control de calidad de los resultados con el SIPQC, cuyo ejemplo se ilustra en la FIGURA 7.

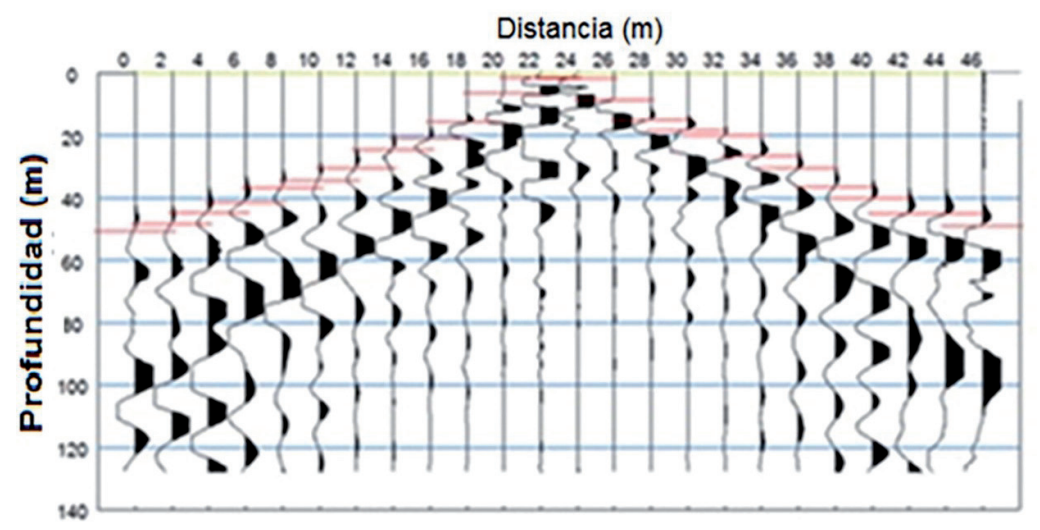

FIGURA 5. Sismograma y marcación de las primeras llegadas con SIPQC. 


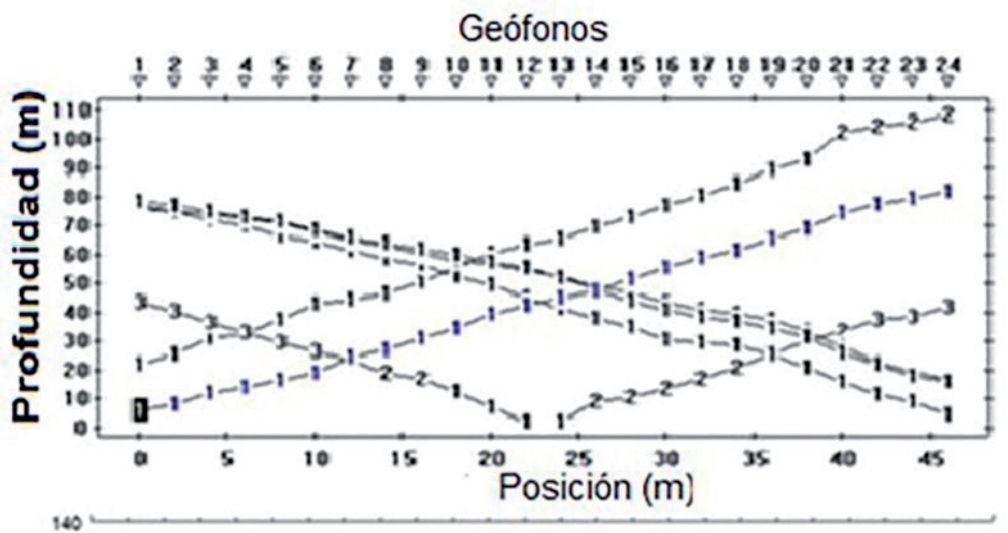

FIGURA 6. Gráfico tiempo-distancia generado con el SIPQC.

\section{Geófonos}

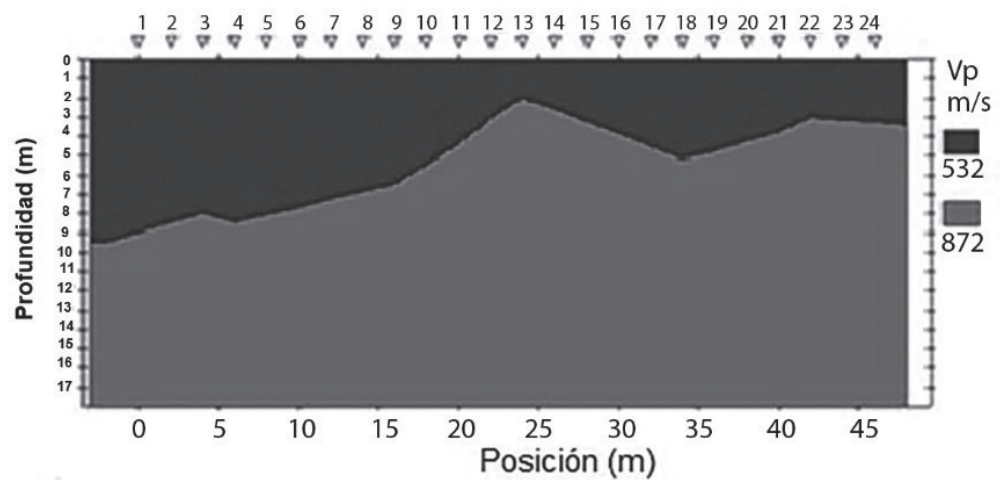

FIGURA 7. Modelo de profundidad generado con SIPQC.

Posteriormente, los datos fueron sometidos al procesamiento final con la utilización del software SEISIMAGER 2D, que incorpora diversos paquetes entre ellos el Pickwin y el Plotrefa versiones 4.4.1.4 y 2.9.2.6, respetivamente, todas de la Geometrics Inc. Para la marcación de las primeras llegadas fue usado el paquete Pickwin, y para la generación de los gráficos de los tiempos de viaje y del modelo de velocidades el Plotrefa. En las FIGURAS 8 y 9 se presentan, respetivamente, el sismograma y el gráfico tiempo-distancia.

\section{Distancia $(m)$}

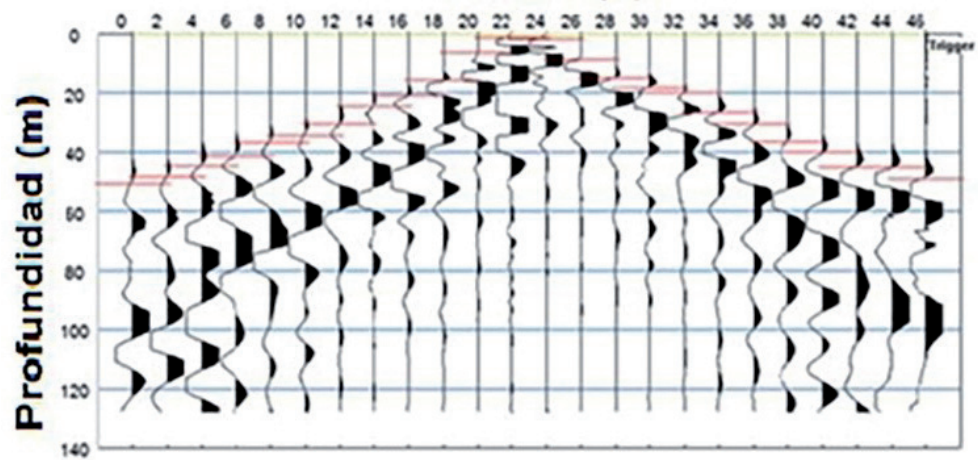

FIGURA 8. Sismograma resultado del punto de impacto en la posición -12 m. 


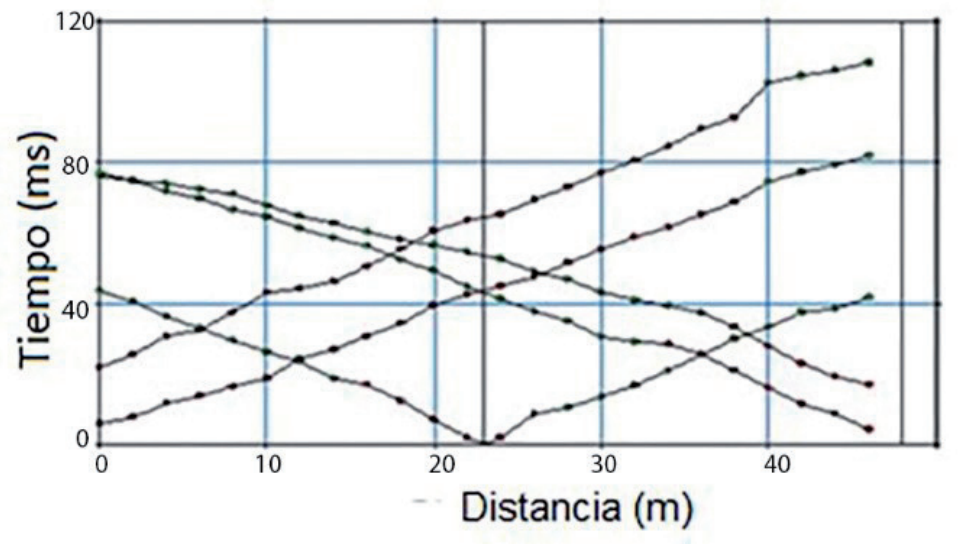

FIGURA 9. Gráfico de los tiempos de viaje.

La FIGURA 10, ilustra el modelo de velocidades de propagación de las ondas sísmicas correspondiente al perfil sísmico. En el modelo pueden ser observadas dos capas con velocidades distintas, siendo el espesor de la segunda capa ilimitada, lo que refleja una situación en la que las interfaces refractoras son irregulares y en que la velocidad aumenta con la profundidad, que es condición necesaria para un levantamiento de sísmica de refracción. Los valores de las velocidades en las capas pueden variar en función de algunos factores como la húmedad local o alteraciones climáticas, y no siempre es posible lograr valores de velocidades idénticos, pero solamente con intervalos pequeños. Se puede verificar que el espesor de la primera capa disminuye significativamente en el punto 30 , mostrando que existe variación lateral del espesor en las capas observadas.

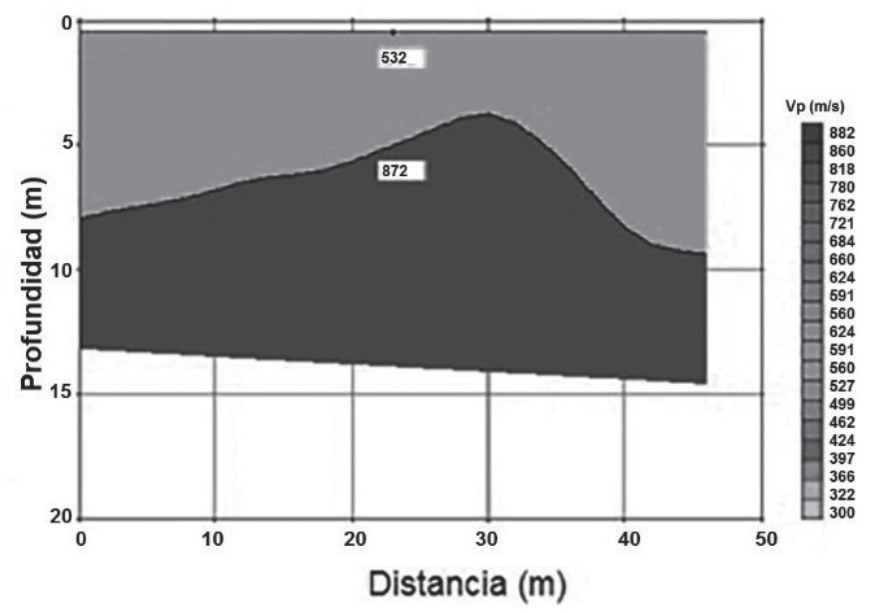

FIGURA 10. Modelo de velocidades para el perfil sísmico Nueva Vida.

\section{RESULTADOS}

La mayor aplicación del método de refracción sísmica en este trabajo fue explorar los efectos geotécnicos someros a profundidades entre 0 y $30 \mathrm{~m}$, permitiendo hacer la caracterización estratigráfica del subsuelo, determinar la profundidad donde el suelo está más compacto, y calcular algunas propiedades físico-mecánicas.
Los datos sísmicos someros fueron procesados usando el programa SeisImager, con el objetivo de estudiar las ondas longitudinales directa y refractada. Como no fue realizada una metodología para estudiar las ondas superficiales, la velocidad de las ondas transversales de la primera capa se estimó con el método 1D MASW utilizando los disparos más apartados del registro con el subsiguiente análisis del carácter dispersivo 
del área. A través del Pickwin fue posible seleccionar los valores de tiempo de las primeras entradas de las ondas $\mathrm{P}$ registradas en cada sismograma del perfil que componen el área, utilizando los datos de refracción. Los resultados fueron interpretados en el módulo Plotrefa donde la interpretación fue realizada utilizando el método Time Term Inversion para dos tres capas y tomografia sísmica.

Los datos logrados de la tomografia sísmica fueron interpolados y representados como puede observarse en la FIGURA 11.

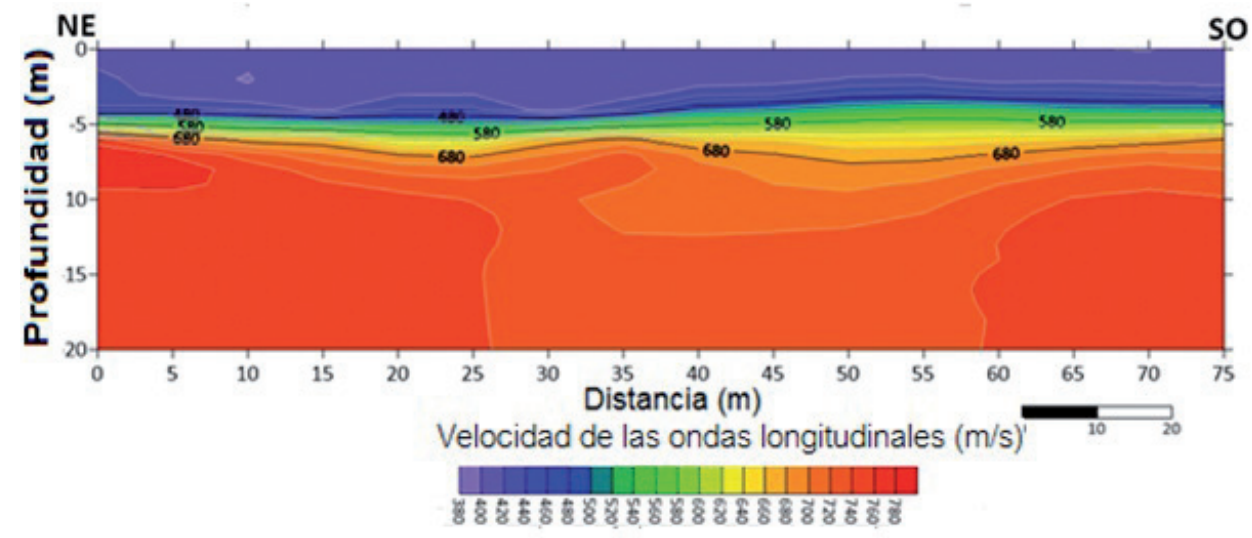

FIGURA 11. Resultado de la tomografia sísmica.

Los valores de velocidad fueron correlacionados con los valores SPT, teniendo en cuenta que los valores de velocidad calculados con la refracción sísmica no coinciden exactamente con la localización de las prospecciones SPT, sin embargo, fue realizado ese análisis para no usar las fórmulas propuestas por otros autores en diferentes condiciones geológicas y geotécnicas.
Cuando la correlación fue realizada con los datos de todos los ensayos SPT (TABLA 2), se obtuvo un coeficiente de correlación lineal de $\mathrm{r}=0,86$ que es aceptable si se tiene en cuenta que las dos variables no fueron medidas en la misma localización exactamente.

TABLA 2. Datos utilizados para realizar la regresión.

\begin{tabular}{|c|c|c|c|c|c|c|c|c|c|}
\hline ENSAYO & $\mathbf{H}(\mathbf{m})$ & $X(m)$ & $\mathbf{N}_{\text {SPT }}$ & $\begin{array}{c}\mathbf{V P} \\
(\mathrm{m} / \mathrm{s}) \\
\end{array}$ & ENSAYO & $\mathbf{H}(\mathbf{m})$ & $X(m)$ & $\mathbf{N}_{\text {SPT }}$ & $\begin{array}{c}\mathbf{V P} \\
(\mathrm{m} / \mathrm{s})\end{array}$ \\
\hline \multirow{10}{*}{ SPT -S3 } & -2 & 10 & 22 & 404,1 & \multirow{4}{*}{ SPT -S1 } & -2 & 37 & 18 & 405,2 \\
\hline & $-3,5$ & 10 & 27 & 404,2 & & $-3,5$ & 37 & 21 & 406,2 \\
\hline & -5 & 10 & 32 & 406,3 & & -5 & 37 & 38 & 468 \\
\hline & $-6,5$ & 10 & 40 & 706,4 & & $-6,5$ & 37 & 60 & 684,5 \\
\hline & -8 & 10 & 50 & 747,7 & \multirow{10}{*}{ SPT -S2 } & -2 & 67,6 & 52 & 410,9 \\
\hline & $-9,5$ & 10 & 60 & 747,7 & & $-3,5$ & 67,6 & 43 & 530,3 \\
\hline & -11 & 10 & 60 & 747,7 & & -5 & 67,6 & 54 & 666 \\
\hline & $-12,5$ & 10 & 60 & 747,7 & & $-6,5$ & 67,6 & 54 & 741,6 \\
\hline & -14 & 10 & 60 & 747,7 & & -8 & 67,6 & 60 & 741,6 \\
\hline & $-15,5$ & 10 & 60 & 747,7 & & $-9,5$ & 67,6 & 60 & 742,1 \\
\hline \multirow{9}{*}{ SPT -S1a } & -2 & 21,2 & 38 & 421 & & -11 & 67,6 & 60 & 742,5 \\
\hline & $-3,5$ & 21,2 & 42 & 425,1 & & $-12,5$ & 67,6 & 60 & 742,5 \\
\hline & -5 & 21,2 & 48 & 631,9 & & -14 & 67,6 & 60 & 742,5 \\
\hline & $-6,5$ & 21,2 & 48 & 745,3 & & $-15,5$ & 67,6 & 60 & 742,5 \\
\hline & -8 & 21,2 & 50 & 746,3 & & \multirow{5}{*}{$\mathrm{r}=0,86$} & & & \\
\hline & $-9,5$ & 21,2 & 60 & 747,3 & & & & & \\
\hline & -11 & 21,2 & 60 & 748,3 & & & & & \\
\hline & $-12,5$ & 21,2 & 60 & 749,3 & & & & & \\
\hline & -14 & 21,2 & 60 & 749,3 & & & & & \\
\hline
\end{tabular}


La ecuación de correlación lograda fue:

$$
N_{S P T}=-0,286+0,0779 V_{P}
$$

Donde $\mathrm{N}_{\mathrm{SPT}}$ es el Número de Golpes de los ensayos de $\mathrm{SPT}$ y $\mathrm{V}_{\mathrm{P}}$ es la velocidad de las ondas sísmicas longitudinales.

La prueba de hipótesis realizada confirma que la ecuación es significativa aunque en algunos puntos los parámetros estimados a partir de ella no fueron muy buenos, sobre todo en el área cercana al ensayo S1, esto puede ser causado por la presencia de fragmentos de calizas dentro de estos suelos arenosos.

Esto prueba que con las velocidades obtenidas con el método de refracción sísmica puede estimarse los valores de $\mathrm{N}_{\mathrm{SPT}}$ Usando la fórmula 1 todos los valores de velocidad $\mathrm{Vp}$ fueron convertidos en valores de $\mathrm{N}_{\mathrm{SPT}}$ como se puede observar en la FIGURA 12.

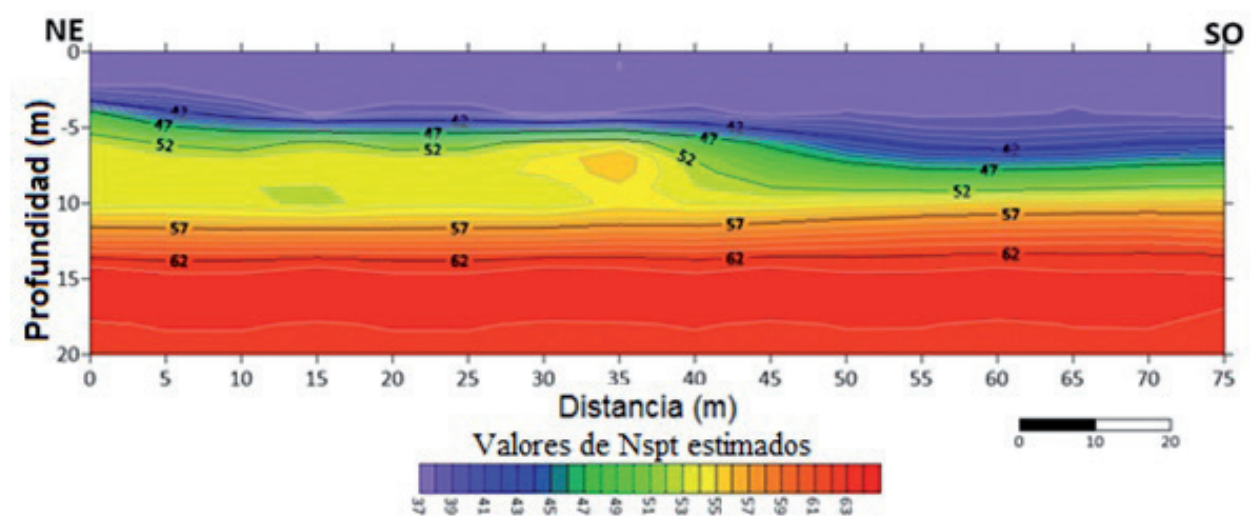

FIGURA 12. Valores $\mathrm{N}_{\mathrm{SPT}}$ estimados desde las velocidades obtenidas por la sísmica de refracción.

Para calcular los parámetros físico-mecánicos fueron utilizados los datos de velocidad longitudinal creado por la tomografia sísmica.

Las velocidades de las ondas transversales fueron estimadas con el software SeisImager empleando los disparos más distantes de la sísmica de refracción e interpretado con la metodología 1D MASW, los resultados son presentados en la FIGURA 13. Se tiene una velocidad constante hasta los 10 metros de profundidad, el valor $\mathrm{Vs}=277 \mathrm{~m} / \mathrm{s}$ puede ser utilizado para el cálculo de las propiedades físico mecánicas.

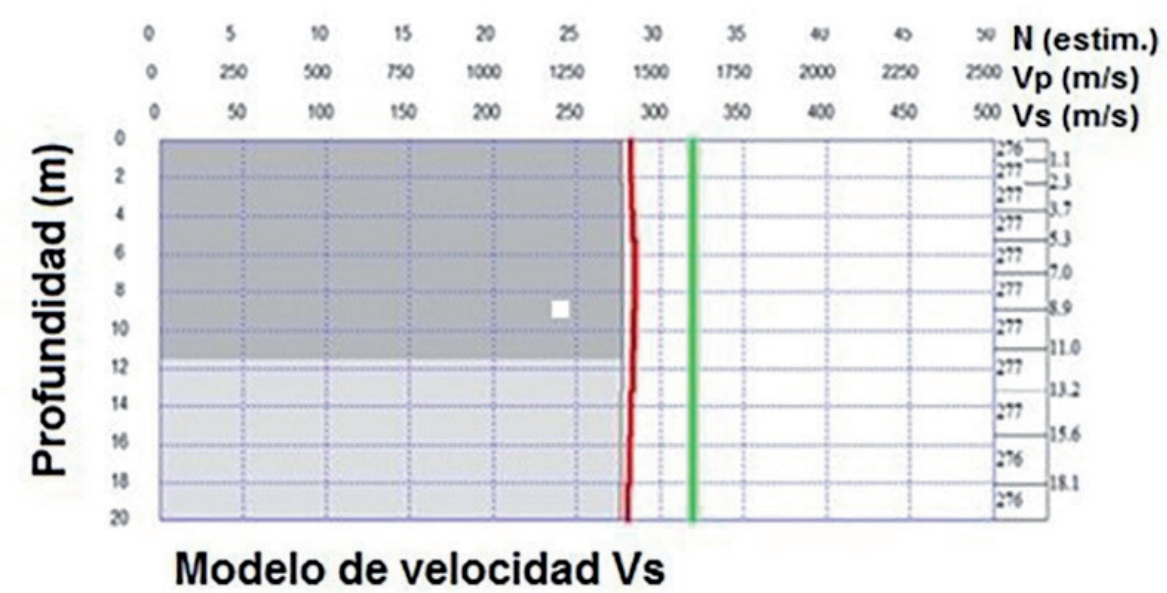

FIGURA 13. Estimativa de las Velocidades transversales (Vs) con el método 1DMASW. 
Una vez obtenido el perfil de velocidades de las ondas longitudinales $(\mathrm{Vp})$ y el de las ondas transversales (Vs) se estimaron otros parámetros geotécnicos que son muy importante para el cálculo de la capacidad portante de los suelos utilizando las siguientes expresiones matemáticas.
Módulo de Elasticidad o Módulo de Young (Edin): Representa la cantidad de esfuerzo por unidad de deformación $\mathrm{E}=\sigma / \varepsilon$.

$$
E_{d i n}=\delta V_{S}^{2}\left(\frac{3 V_{P}^{2}-4 V_{S}^{2}}{V_{P}^{2}-V_{S}^{2}}\right)
$$

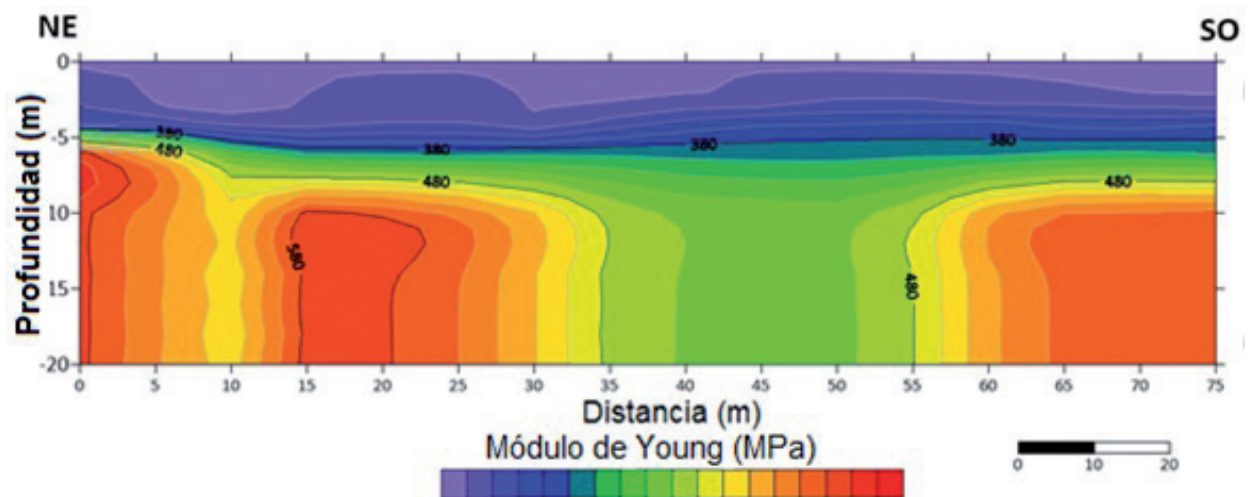

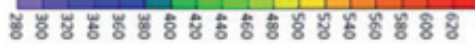

FIGURA 14. Módulo de Young, dinámico calculado a partir de los datos sísmicos.

Módulo de Corte o Cizallamiento (G): definido como la relación entre la tensión y la deformación de corte, es una medida de la dureza del material.

$$
G=\frac{E}{2(1+\eta)}=\delta V_{S}^{2} ; \quad \delta=0,2 V_{P}^{0,25}
$$

(Densidad del material del terreno)

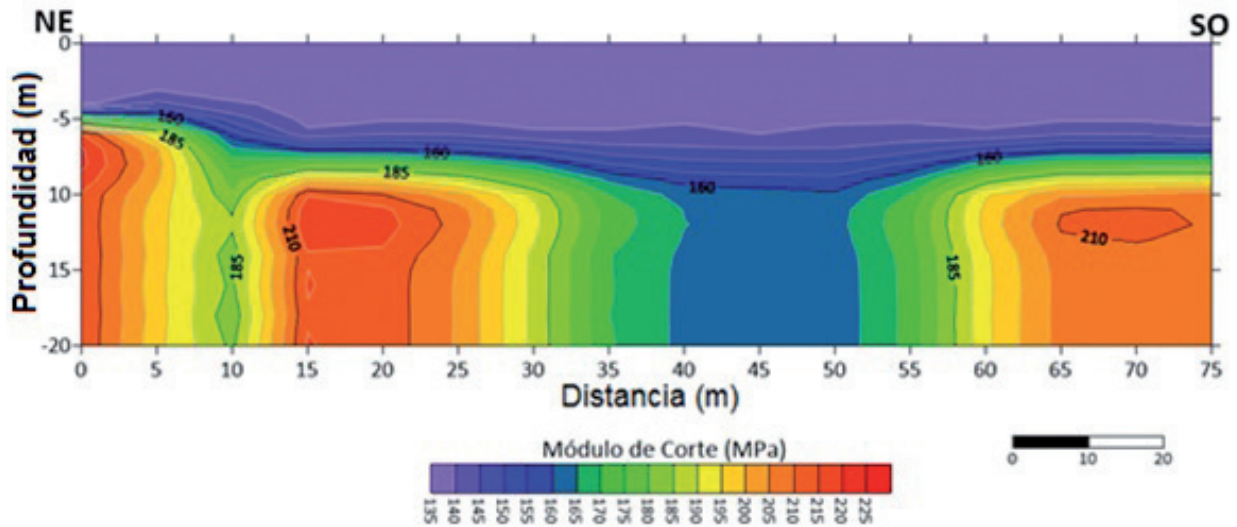

FIGURA 15. Módulo de Corte (G) en MPa, calculado por datos sísmicos.

Coeficiente de Poisson, $\eta$ : Expresa la relación entre las deformaciones transversales y longitudinales.

$$
\eta=\frac{V_{P}^{2}-2 V_{S}^{2}}{2\left(V_{P}^{2}-V_{S}^{2}\right)}
$$




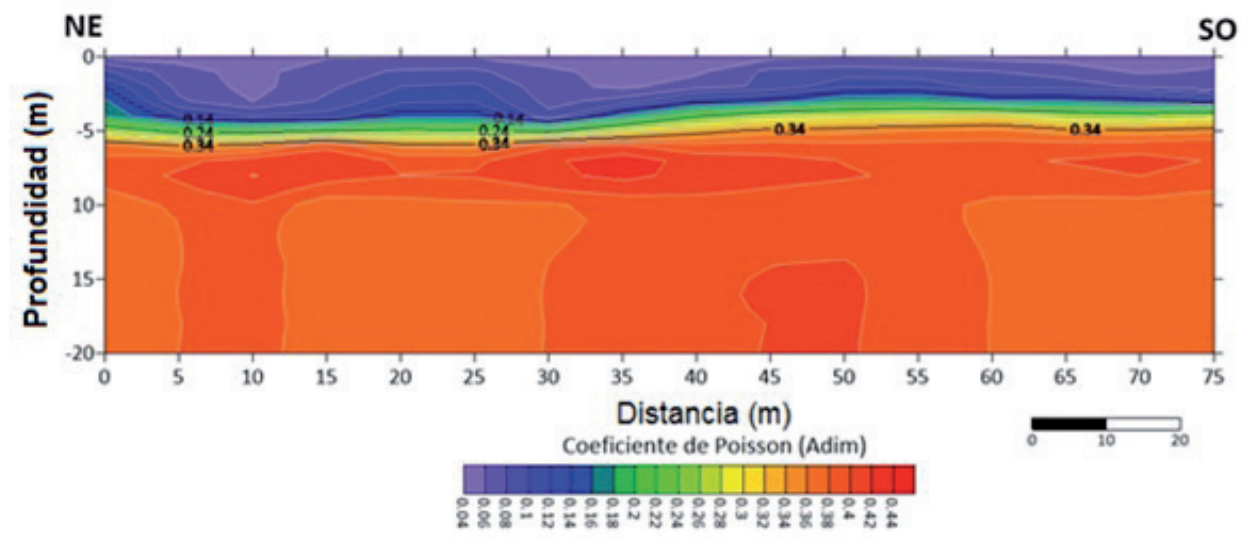

FIGURA 16. Coeficiente de Poisson $(\mu)$ estimado por datos sísmicos.

\section{DISCUSIÓN}

Se observa que las ecuaciones de regresión logradas entre los valores de velocidades y la Resistencia a la Penetración Estándar de los ensayos SPT son significativos, pudiendo ser empleadas con confiabilidad para estimar de manera preliminar este parámetro geotécnico desde la sísmica de refracción. Una gran ventaja que ofrece la sísmica es que se obtienen datos más continuos que los ofrecidos por las prospecciones geotécnicas.

Por la descripción litológica de las prospecciones se observa la presencia de rocas calizas en el centro del área coincidente con la prospección S1, éste tuvo que ser eliminado del análisis porque ya a los 5 metros no se pudo continuar con el ensayo de penetración estandar. En estos suelos es común que aparezcan fragmentos de roca caliza dentro de las capas. Cuando se realizó el perfil con los datos de $\mathrm{N}_{\mathrm{SPT}}$ sin corregir, se observó como en esa zona aumentaba estos valores a poca profundidad (FIGURA 3), la información sísmica corroboró igualmente esta situación al existir un aumento de las velocidades sísmicas en esta zona (FIGURA 12), se puede ver que con la sísmica se detallan las dimensiones de este cuerpo, la presencia de fragmentos de rocas calizas pueden afectar los ensayos geotécnicos, dando lugar a falsas interpretaciones y pensar que se encontró un suelo más compacto para las cimentaciones, cuando no es así. En este trabajo se decidió hacer otro ensayo de SPT a pocos metros y no se encontraron los fragmentos de rocas.

Para calcular las velocidades de las ondas transversales se utilizó el disparo más distante $(6 \mathrm{~m})$, con el objetivo de interpretarlo utilizando la metodología 1D MASW, esto no es lo correcto y solo se logra una velocidad con la profundidad como puede ser observardo en la FIGURA 13. Lo ideal es realizar estudios de ondas superficiales utilizando las metodologías de adquisición de los métodos 1D MASW, 2D MASW o REMI descritos por diferentes autores (Humire et al., 2015), todos estos procedimientos permiten calcular las velocidades de las ondas transversales con mayor precisión.

En las FIGURAS 14, 15, y 16 se observa como el módulo de Young, módulo de Rigidez y el coeficiente de Poisson aumentan con la profundidad delimitando dos capas, por otro lado puede observarse, como en el lugar donde existía presencia de fragmentos de rocas calcáreas, se presentan los valores mas bajos de los modulos de Young y de Corte, indicando condiciones geotécnicas más mala, es posible que en esta área se hubiese realizado algún tipo de excavación, de ahí que los suelos esten menos compactos y que existan fragmentos de rocas.

\section{CONCLUSIONES}

La presentación de los parámetros geotécnicos en mapas 2D, son más representativos que la información tabulada.

Se puede convertir todos los valores de velocidades en parámetros geotécnicos si existen ecuaciones de correlaciones lo suficientemente significativas.

Al tener las velocidades de las ondas longitudinales y las transversales se pueden estimar mucho más parámetros geotécnicos y representarlos en mapas 2D.

En el áea de estudio se observó refracciones debiles, debido a que no existen grandes contrastes entre las velocidades de las diferentes capas de arena, limo y 
arcillas con la profundidad, causados principalmente por la ausencia de un sustrato rocoso.

Se observó un nivel de ruido alto en el sismograma obtenido propio del ambiente urbano donde se hicieron las mediciones sísmicas.

\section{AGRADECIMIENTO}

Los autores agradecen a SASSCAL (Southern African Science Service Centre Sea Climate Change and Adaptive Land Use) por la financiación del equipo y algunas actividades para el desarrollo de este trabajo.

\section{REFERENCIAS}

Araújo, P., y Peres-Rocha, M. (2010). Estudo do método geofísico de sísmica de refração com aplicação no futuro trecho norte do metrô de Brasília. Curso de Física, Universidade Católica de Brasília e Instituto de Geociências - Universidade de Brasília.

ASTM D5777-95. 1999. Standard Guide for Using the Seismic Refraction Method for Subsurface Investigation.

Humire, F., Sáez, E., y Leyton, F. (2015). Manual de aplicación de técnicas geofísicas basadas en ondas de superficie para la determinación del parámetro VS30. Santiago de Chile: Instituto de la Construcción.

Lankston, R.W. (1989). The seismic refraction method: A viable tool for mapping shallow targets into the 1990s. Geophysics, 54, 1535-1542.

Luna, R., and Jadi, H. (2000). Determination of dynamic soil properties using geophysical methods. First International Conference on the Application of Geophysical and NDT Methodologies to Transportation Facilities and Infrastructure. St. Louis, USA.
Nabil, H.A., and Al-Heety, A.J. (2014). Seismic refraction tomography and MASW survey for geotechnical evaluation of soil for the teaching hospital project at Mosul University. Journal of Zankoy Sulaimani- Part A, 16(1), 1-14. doi: 10.17656/jzs.10279.

Naranjo, H., y Dranichnikov, T. (2012). Cálculo de capacidad portante basado en geofísica y método convencional. El caso del puente sobre $o$ rio Maicito. Quito: Universidad Politécnica Salesiana.

Sacasas, C. (2012). Sísmica Somera. Notas de Clases. Cujae. La Habana.

Tecnasol. (2007). Edificio No Projecto Nova Vida Luanda - Angola Prospecção GeológicoGeotécnica Nota Técnica, Luanda, Dezembro de 2007.

Teixeira Pires, G.J.P. (2006). Caracterização geológica e geotécnica dos solos de Luanda para o ordenamento do território. Tese de Mestrado, Departamento de Geología, Universidade de Lisboa, Portugal.

Maria Amélia Pereira de Barros da Silva ORCID: 0000-0001-7739-8174

Héctor Manuel Fernández ORCID: 0000-0003-0096-2894

João Júnior Baptista

ORCID: 0000-0001-8888-6923

Gabriela Jeremias Pereira Teixeira-Pires

ORCID:0000-0002-6130-3959

Trabajo recibido: septiembre 17 de 2017

Trabajo aceptado: mayo 03 de 2018 